\title{
Funções Básicas da HP 50G para Engenharia
}

\author{
Bruna Sayuri de Souza Suzuki, Éber Saj Porcacchia
}

\begin{abstract}
Resumo - Esse material foi preparado e distribuído para os participantes do Minicurso de HP 50G realizado pelo PET Mecatrônica nos dias 28 de setembro de 2017 e 05 de outubro de 2017. Para a publicação na revista, foram acrescentados mais alguns tópicos. O Minicurso de HP 50G focou principalmente nos alunos do quarto semestre do curso de Engenharia Mecatrônica da Escola Politécnica da USP. Nesse semestre, em especial para as disciplinas de Mecânica dos Fluidos, Eletricidade Geral e Estatística, a utilização de uma calculadora gráfica é altamente recomendável para a resolução de exercícios. Devido ao fato de a HP 50G ser uma calculadora bastante completa, que atende a todas as necessidades e já bastante utilizada no meio politécnico, realizamos esse minicurso para que os alunos conhecessem as principais funções utilizadas na HP nessas disciplinas.
\end{abstract}

Palavras-chave - Calculadora gráfica; Minicurso; HP50G

\section{Title - HP 50G Basic Functions for Engineering}

\begin{abstract}
This material was prepared and distributed to the participants of the HP 50G Minicourse, organized by PET Mecatrônica in September 28th, 2017 and October 5th, 2017. For this publication, some topics were added. The HP 50G Minicourse targeted mainly the fourth semester students of Mecathronic Engineering of Escola Politécnica da USP. In this semester, specially in Fluid Mechanics, Electricity and Statistics, a graphic calculator is highly recommended to be used to solve exercises. Due to the fact that HP 50G is very complete, meets the needs of the Engineering course and has already been used by other Poli students, we did this minicourse in order to show some important functions that are used in these subjects.
\end{abstract}

Keywords - Graphic calculator; Minicourse; HP50G

Bruna Sayuri de Souza Suzuki cursa Engenharia Mecatrônica desde 2015 e, nesse mesmo ano, se tornou membro do PET Mecatrônica. Foi também Editora-Chefe da revista Mecatrone durante o ano de 2017.

Éber Saj Porcacchia cursa Engenharia Mecatrônica desde 2015 e se tornou membro do PET Mecatrônica em 2017. 


\section{MINICURSO de HP 50g}

\section{Configuracões iniciais}

Clicando no botão MODE da HP, aparecerão algumas opções.

\section{$\Rightarrow$ Operating Mode}

Modo de operação da calculadora. É possível escolher entre o algébrico e o de pilha.

\section{$\Rightarrow$ Number Format}

Formato do número. Standard é o padrão, no qual o número é mostrado com um arredondamento de 11 casas decimais. Em Fixed, é possível escolher o número de casas decimais, além de serem mostrados os milhares sendo separados por vírgula. Scientific mostra o número por meio de notação científica de acordo com o número de casas decimais escolhido, e Engineering irá mostrar em notação científica, no qual os expoentes de 10 são múltiplos de 3 , e o número escolhido é o número de casas decimais mostrados.

\section{$\Rightarrow$ Angle Measure}

Escolha de medida do ângulo, que pode ser em graus, radianos ou grados (unidade de medida de ângulos planos equivalente a $\pi / 200$ do radiano ou $9 / 10$ do grau, ou seja, a $1 / 400$ de uma rotação completa (revolução)).

\section{$\Rightarrow$ Coord System}

Sistema de coordenadas adotado. É possível escolher entre Retangular, Polar e Esférico.

Algumas opções marcáveis:

FM: mudar para vírgula o separador fracional

Beep: tirar/colocar o som da calculadora

Clicando-se em CAS, recomenda-se deixar marcadas as opções "Complex", habilitando o cálculo de números complexos, e "Approx", para os números serem mostrados de modo numérico decimal. Caso prefira ter os resultados em forma de fração, não habilite essa função. Nesse caso, é possível clicar SHIFT Laranja + ENTER para obter os resultados de forma aproximada toda vez que for realizar os cálculos.

Não se esqueça de clicar em OK sempre depois de alguma alteração nas configurações para ser ativada.

\section{$\underline{\text { Ajustando Data e Hora }}$}

Primeiramente, clique no botão MODE e vá em DISP na tela. Marque a opção Clock. Após isso, dê OK duas vezes e clique nas teclas SHIFT Laranja + 9, acessando o menu TIME. Selecione a opção 3, "Set time, date". Aqui, é possível arrumar o horário, a data e mudar o modo de exibição de ambos. Com isso, a HP passará a mostrá-los na tela inicial no canto superior direito. 


\section{$\underline{\text { Resolver uma equação numericamente }}$}

A HP50G é muito útil para se resolver equações em que é impossível ou muito difícil de se isolar a incógnita de interesse. Como, por exemplo, a equação abaixo:

$$
2 x-\cos (x)=0
$$

Para resolvê-la na HP50G, abra o menu NUM.SLV (SHIFT Laranja + 7) e siga os seguintes passos:

1. "Solve equation.."

2. No campo "Eq:" digite entre aspas simples a expressão matemática a ser resolvida. Nesse caso, atribuiremos o valor da equação a uma variável $\mathrm{Y}$.

$$
\text { ' } \mathrm{Y}=2 * \mathrm{X}-\mathrm{COS}(\mathrm{X}) \text { ' }
$$

3. Aperte OK. Surgirão campos para digitar valores para as incógnitas $X$ ou $Y$. No nosso caso, queremos resolver a equação para $\mathrm{X}$ quando $\mathrm{Y}=0$.

4. Com o campo Y selecionado, aperte EDIT. Insira o valor 0 e aperte OK.

5. Com o campo X selecionado, aperte SOLVE.

Como esperado, a HP50G nos dará a solução da equação, que é $X=0,45018$.

\section{$\underline{\text { Resolvendo um sistema linear } \mathrm{A}^{*} \mathrm{X}=\mathrm{B}}$}

Um sistema linear do tipo

$$
\left\{\begin{array}{l}
x+2 y=10 \\
4 x+9 y=4
\end{array}\right.
$$

Pode ser escrito na forma de matrizes

$$
\left(\begin{array}{ll}
1 & 2 \\
4 & 9
\end{array}\right) *\left(\begin{array}{l}
x \\
y
\end{array}\right)=\left(\begin{array}{c}
10 \\
4
\end{array}\right)
$$

Sendo

$$
A=\left(\begin{array}{ll}
1 & 2 \\
4 & 9
\end{array}\right) \quad e \quad B=\left(\begin{array}{c}
10 \\
4
\end{array}\right)
$$

1. Abra o menu NUM.SLV (SHIFT Laranja + 7), clique em "4. Solve lin sys.." e complete os campos com as matrizes A e B, utilizando o MRTW (SHIFT branco + aspas simples (')).

2. Tecle SOLVE: os valores de $\mathrm{x}$ e y serão dados em forma de matriz coluna.

\section{Números complexos na HP50G}

No menu MODE: utilizaremos o modo RPN, ângulos em degrees, sistema de coordenadas retangular. No CAS dentro do MODE, Approx ativado (representa os resultados em resultado numérico decimal), Complex ativado (permite trabalhar com números complexos).

\section{$\Rightarrow$ Forma retangular}

Para representar um número complexo $a+b i$ em sua forma retangular, é utilizada a notação de par ordenado $(a, b)$, onde $a$ é a parte real e $b$ é a parte imaginária. Os parênteses são acessados com SHIFT Branco + Operador menos (-). A vírgula é acessada com SHIFT Laranja + SPC.

- Soma: Coloque ambos números na pilha. Aperte o botão de soma.

- Multiplicação: Coloque ambos números na pilha. Aperte o botão de multiplicação.

- Divisão: Coloque o numerador na pilha. Coloque o denominador na pilha. Aperte o botão de divisão. 


\section{$\Rightarrow$ Forma polar}

Para representar um número complexo $a+b i$ em sua forma polar, é utilizada a notação $A \angle \theta$, onde $A=\sqrt{a^{2}+b^{2}}$ é o módulo e $\theta=\arctan \left(\frac{b}{a}\right)$ é o argumento. Na HP, a forma polar é escrita como $(A, \angle \theta)$. Os parênteses são acessados com SHIFT Branco + Operador menos (-). O símbolo $\angle$ é acessado com ALPHA + SHIFT Laranja +6.

As operações básicas são realizadas da mesma maneira descrita anteriormente.

\section{$\Rightarrow$ Sistemas lineares com números complexos}

$\mathrm{Na}$ disciplina de Eletricidade Geral, é comum a resolução de sistemas lineares em que os coeficientes são números complexos com parte imaginária não nula. Utilizar a HP facilita muito esse trabalho.

1. Como visto anteriormente, para inserirmos um sistema linear, vamos em NUM.SLV (SHIFT Laranja $+7)$, seguido de "4.Solve lin sys..".

$$
\left\{\begin{array}{c}
\mathrm{x}_{1}+\mathrm{x}_{3}=54,3 \angle 89,8^{\circ} \\
\mathrm{x}_{1}+\mathrm{x}_{2}-\mathrm{x}_{3}=0 \\
(1+0,4 i) \mathrm{x}_{1}+(3-1,6 i) \mathrm{x}_{2}=141,4 \angle-90^{\circ}
\end{array}\right.
$$

2. Preencheremos agora as matrizes A e B. O procedimento é semelhante ao anterior, devendo-se agora somente tomar um cuidado maior no formato dos números inseridos.

$$
A=\left(\begin{array}{ccc}
1 & 0 & 1 \\
1 & 1 & -1 \\
1+0,4 i & 3-1,6 i & 0
\end{array}\right) \quad B=\left(\begin{array}{c}
54,3 \angle 89,8^{\circ} \\
0 \\
141,4 \angle-90^{\circ}
\end{array}\right)
$$

3. Todos os números, inclusive aqueles com parte imaginária nula, devem ser escritos na forma de par ordenado. Para isso, basta escrever o primeiro nesse formato e os próximos serão convertidos automaticamente pela HP.

4. Com o campo X selecionado, aperte SOLVE.

$$
\begin{gathered}
\mathrm{x}_{1}=-31,06+28,15 i=41,92 \angle 137,81^{\circ} \\
\mathrm{x}_{2}=-62,31+2,01 i=62,34 \angle-1,85^{\circ} \\
\mathrm{x}_{3}=31,25+26,14 i=40,74 \angle 39,92^{\circ}
\end{gathered}
$$

5. Se você desejar visualizar os resultados na forma polar, altere para coordenadas polares em MODE $>$ Coord System.

OBSERVAÇÃO: A HP não aceita raízes dentro da forma polar. Por exemplo, colocar o número $\left(\sqrt{3}, \angle 80^{\circ}\right)$ resulta em sintaxe inválida. Com isso, devemos fazer $\sqrt{3}\left(1, \angle 80^{\circ}\right)$ para que a HP aceite. Não é necessário colocar a vírgula entre 1 e $\angle 80^{\circ}$, ou seja, colocar $\sqrt{3}\left(1 \angle 80^{\circ}\right)$ já é o suficiente.

\section{$\underline{\text { Salvar uma equação }}$}

Estando com o modo RPN ativado, para salvar uma equação dentro da HP50G, siga os seguintes passos:

1. Abra o Equation Writer, que é acessado por SHIFT Laranja + aspas simples ('). Essa opção é muito útil para facilitar na escrita de equações complexas.

2. Digite a equação que se deseja salvar, por exemplo, a equação de Colebrook de Mecânica dos Fluidos. 


$$
\frac{1}{\sqrt{f}}=-2,0 \log \left(\frac{\epsilon}{3,7 D}+\frac{2,51}{\operatorname{Re} \sqrt{f}}\right)
$$

3. Aperte ENTER e a expressão será adicionada à pilha.

4. Com o modo Alpha ativado, digite o nome da equação (por exemplo, COLEBROOK) e aperte ENTER para também adicioná-lo a pilha. (Dica: clique 2 vezes no ALPHA para travar o modo alfanumérico).

5. Aperte o botão STO> e a equação será salva na HP50G dentro da variável com o nome digitado.

6. Agora, quando quisermos resolver a equação salva, devemos ir para o menu NUM.SLV (SHIFT Laranja + 7), e em seguida "1. Solve equation..".

7. No campo "Eq:", aperte CHOOS e selecione na lista de equações salvas dentro da HP qual você deseja solucionar. No nosso caso, 'EQ'COLEBROOK.

8. Preencha os valores das variáveis conhecidas e, em seguida, aperte SOLVE no campo da variável que se deseja descobrir. Por exemplo:

$$
\epsilon=0,00015 \quad R e=275000 \quad D=0,09
$$

9. Obtém-se o resultado $f=0,023$

Quando for necessário apagar uma equação que foi salva em uma variável dentro da HP50G, devemse seguir os passos:

1. Coloca-se na pilha o nome da equação entre chaves. No nosso caso, \{COLEBROOK\}.

2. Em seguida, com o menu TOOL ativado, aperte o botão PURGE (F5).

3. Pronto, a variável COLEBROOK foi liberada.

4. É possível realizar esse processo para mais de uma variável de uma vez, separando-as por vírgulas dentro das chaves \{COLEBROOK, TAYLOR, MAXWELL\}.

Para verificar as variáveis e equações que estão na memória da HP, clique SHIFT Branco + APPS, acessando o menu FILES. Dentro da opção Home, será mostrado tudo o que está sendo guardado na memória. É possível selecionar alguma variável ou equação para visualizar e/ou editar. Caso queira apagá-la, clique na tecla NXT e selecione a opção PURGE que aparecerá na tela.

\section{$\underline{\text { Estatística }}$}

Com certeza você usará bastante a HP para cálculos de dados estatísticos. Vamos ver a seguir as funções mais utilizadas.

Primeiramente, abra o menu STAT (SHIFT Laranja + 5) e selecione:

\section{$\Rightarrow$ Single-var.}

Aqui será possível realizar cálculos de média aritmética (Mean), desvio padrão (Std Dev), variância (Variance), soma total dos valores, valor mínimo e valor máximo dentre os colocados na tabela.

Em $\sum D A T$, insira os valores que você queira para realizar os cálculos. Você pode colocar EDIT ou então MTRW (SHIFT branco + aspas simples (')) para escrever uma nova matriz. Observe que nesse caso, a HP só irá realizar os cálculos com os valores colocados na COLUNA 1 da tabela (podendo ser mudado no campo COL).

Por exemplo, queremos os cálculos dos valores:

\begin{tabular}{|l|l|l|l|l|l|}
\hline 7 & 2 & 0.5 & 4 & 10 & 1.5 \\
\hline
\end{tabular}

1- Escreva esses valores na coluna 1 da matriz.

2- Selecione os resultados que queira obter e clique em OK.

Como esperado, obtemos:

Mean: 4.16666666667 
Total: 25

Maximum: 10

Minimum: 0.5

\section{$\Rightarrow$ Summary Stats}

Muitas vezes, como em ANOVA, precisamos obter a somatória de vários conjuntos de valores. Aqui será possível obter:

$\sum X=$ somatória de todos os valores da $\mathrm{X}-\mathrm{Col}$

$\sum Y=$ somatória de todos os valores da Y-Col

$\sum X 2=$ somatória de todos os valores da coluna $X$, cada valor elevado ao quadrado

$\sum Y 2=$ somatória de todos os valores da coluna $\mathrm{Y}$, cada valor elevado ao quadrado

$\sum X Y=$ somatória de cada $\mathrm{X}$ multiplicado por seu correspondente $\mathrm{Y}$

$\mathrm{N} \Sigma=$ contagem do número de linhas

Tomando como exemplo a tabela abaixo:

\begin{tabular}{|l|l|}
\hline$x$ & $y$ \\
\hline 1 & 10 \\
\hline 2 & 13 \\
\hline 3 & 15 \\
\hline 4 & 14 \\
\hline 5 & 17 \\
\hline 6 & 16 \\
\hline
\end{tabular}

1. Escreva essas valores na matriz,

2. Verifique se $\mathrm{X}-\mathrm{Col}$ e $\mathrm{Y}-\mathrm{Col}$ estão corretos,

3. Selecione os resultados que queira obter e clique em OK.

Como esperado, os resultados são:

$\sum X: 21$

$\sum Y: 85$

$\sum X 2: 91$

$\sum Y 2: 1235$

$\sum X Y: 318$

$\mathrm{N} \sum: 6$

\section{$\Rightarrow$ Fit data}

Aqui é possível obter regressão, correlação e covariância dos valores colocados. Muito útil para a interpolação de pontos.

Exemplo: Temos a seguinte tabela:

E queremos o valor de $\mathrm{y}$ para $\mathrm{x}=281$.

Para tal, siga os passos abaixo.

\begin{tabular}{|l|l|}
\hline$x$ & $y$ \\
\hline 250 & 22,3 \\
\hline 300 & 26,3 \\
\hline
\end{tabular}

1- Em $\Sigma$ DAT, coloque os valores na matriz e dê OK,

2- Defina qual é a coluna dos valores de $\mathrm{X}$ e qual é a dos valores de $\mathrm{Y}$,

3- Deixe "Linear Fit" para regressão linear,

4- Clique em PRED para a calculadora "prever" qual será o valor,

5- Coloque um valor de $\mathrm{X}$ ou de $\mathrm{Y}$ que já possua (nesse caso, temos o valor de 281 para X),

6- Clique em PRED.

Resultado: 24,78 . 
Dicas gerais:

- Sempre que o menu de opções da tela inicial estiver diferente, com outras opções, clique no botão TOOL para que o menu volte ao normal.

- Acesse o game tetris na HP! Para tal, acesse a opção Equation Writer (SHIFT Laranja + aspas simples (')) e digite MINEISBETTER utilizando as letras acessadas pela tecla ALPHA amarela. Após isso, selecione a palavra inteira clicando a tecla de seta para cima da HP, e acesse a opção SIMP que aparece na tela. Pronto! Utilize os números 4, 6 e 2 para mover as peças e 5 para rotacioná-las. Pra sair do jogo, clique na tecla de apagar (com um desenho de uma seta para esquerda) para ver sua pontuação e sair.

- Acesse o campo minado na HP! Clique no botão APPS da HP e selecione a opção Equation Library. Acesse a opção UTILS que aparecerá na tela e depois em MINEH. Aparecerá uma função "MINEHUNT( )". Clique no botão ENTER. Agora sua missão será chegar até o lado direito sem encostar em nenhuma bomba! Utilize as teclas dos números $8,2,4,6,7,9,1$ e 3 para mover para cima, baixo, esquerda, direita e as respectivas diagonais. 